\title{
STK899704 inhibits stemness of cancer stem cells and migration via the FAK-MEK-ERK pathway in HT29 cells
}

\author{
Hui-Ju Jang ${ }^{1}$, Yesol Bak ${ }^{1}$, Thu-Huyen Pham ${ }^{1}$, Sae-Bom Kwon ${ }^{1}$, Bo-Yeon Kim ${ }^{2}$, JinTae Hong ${ }^{3}$ E Do-Young Yoon ${ }^{1, *}$ \\ ${ }^{1}$ Department of Bioscience and Biotechnology, Konkuk University, Seoul 05029, ${ }^{2}$ World Class Institute, Anticancer Agents Research \\ Center, Korea Research Institute of Bioscience and Biotechnology, Chungbuk 28116, ${ }^{3}$ College of Pharmacy and Medical Research Center, \\ Chungbuk National University, Cheongju 28160, Korea
}

Colon cancer is one of the most lethal and common malignancies worldwide. STK899704, a novel synthetic agent, has been reported to exhibit anticancer effects towards numerous cancer cells. However, the effect of STK899704 on the biological properties of colon cancer, including cancer cell migration and cancer stem cells (CSCs), remains unknown. Here, we examined the inhibitory effect of STK899704 on cell migration and CSC stemness. In the wound healing assay, STK899704 significantly inhibited the motility of colon cancer cells. Furthermore, STK899704 downregulated the mRNA expression levels of the cell migration mediator focal adhesion kinase (FAK). STK899704 also suppressed mitogen-activated protein kinase kinase and extracellular signal-regulated kinase, which are downstream signaling molecules of FAK. Additionally, STK899704 inhibited stemness gene expression and sphere formation in colon cancer stem cells. These results suggest that STK899704 can be used to treat human colon cancer. [BMB Reports 2018; 51(11): 596-601]

\section{INTRODUCTION}

Colon cancer is the second most common cause of cancer-related deaths, with over 1 million people diagnosed annually. The mortality rate associated with colon cancer increased from 490,000 in 1990 to approximately 715,000 in $2010(1,2)$. The lifespan risk of progressing colon cancer is $5.1 \%(3,4)$ and more than $65 \%$ of cases occur in developed countries (5). Subsets of cancer cells such as cancer stem cells (CSCs) are important in cancer recurrence and metastasis. CSCs were first identified in acute myelogenous leukemia (6)

${ }^{*}$ Corresponding author. Tel: +82-2-450-4119; Fax: +82-2-4444218; E-mail: ydy4218@konkuk.ac.kr

https://doi.org/10.5483/BMBRep.2018.51.11.180

Received 6 August 2018, Revised 16 August 2018, Accepted 20 September 2018

Keywords: Cancer stem cell, Colon cancer, Focal adhesion kinase, Migration, STK899704 as a rare subpopulation with high tumor-initiation capacity in bulk tumors (7). CSCs possess the capacities for differentiation into different cell types and self-renewal, as observed in normal adult stem cells (8). As a result, CSCs are thought to play an important role in cancer aggressiveness (9). Additionally, CSCs are associated with metastatic spread from the primary tumor and are known to be resistant to different anti-cancer therapies (10). Current therapies eliminate the bulk of differentiated cancer cells, but fail to kill CSCs, which are conserved by individual resistance strategies (11). Surviving CSCs generate new tumors and initiate metastasis, resulting in disease recurrence. Therefore, studies of CSCs may facilitate the development of therapeutic strategies to improve survival rates. Microtubules are associated with the transport of vesicles, development of cell shape, cell signaling, and microtubule polymerization, which are involved in cell motility (12). Several drugs targeting microtubule dynamics were shown to have anticancer effects (13). Recent studies identified a new synthetic compound, ethyl\{2-methyl-3-[2(naphtho[2,1- $\beta]$ furan-2-ylcarbonyl) carbo hydrazonoyl] $-1 \mathrm{H}$ indol-1-yl\} acetate (named as STK899704; PubChem CID: 5455708), which was generated as a microtubule-depolymerizing agent (14), but its effects on colon cancer cells and colon CSCs are unknown.

In the present study, we investigated the effects of STK899704 on the migration of colon cancer cells and properties of colon CSCs. Our data shows that STK899704 treatment can reduce the migration ability of colon cancer cells by inhibiting migration-related molecules and suppressing the expression of stemness markers in colon CSCs, suggesting that it can be used as a novel strategy for treating colon cancer.

\section{RESULTS}

Cytotoxicity of STK899704 on colon cancer cells and normal colon cells

The previous studies revealed that STK899704 has anticancer effect on lung cancer, cervical cancer and skin cancer (14). First, we investigated the cytotoxic effect of STK899704 at various concentrations $(0.1-0.4 \mu \mathrm{M})$ against numerous cell lines by performing an MTS assay. As shown in Fig. 1B and 


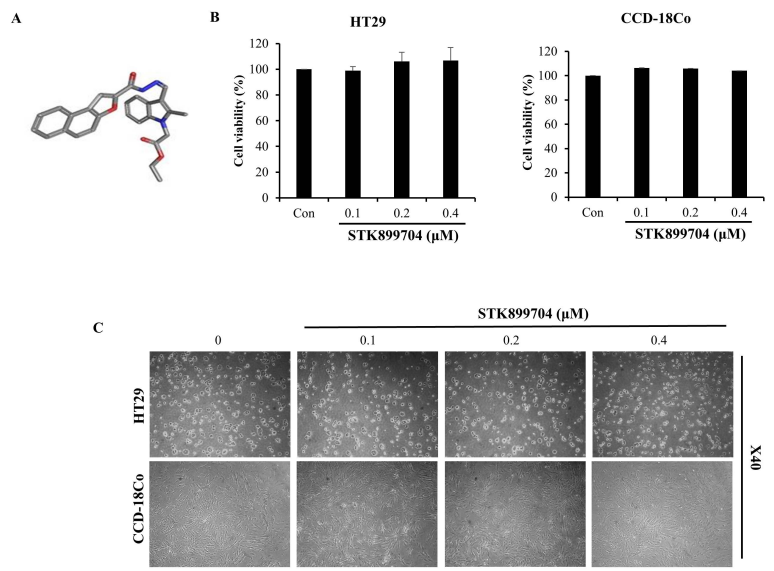

Fig. 1. Effects of STK899704 on cell viability of HT29 colon cancer cells. (A) Structure of STK899704. (B) Viability of HT29 and CCD18-Co cells treated with STK899704 was assessed using an MTS assay. (C) Microscopic images of HT29 cells and CCD18-Co cells treated with STK899704 for $24 \mathrm{~h}$.

Supplemental Fig. 1, the cell viability of HT29 cells was not affected by treatment with STK899704 for up to $96 \mathrm{~h}$. In the normal colon fibroblast cell line CCD-18Co, cell viability was maintained at over $90 \%$ after $24 \mathrm{~h}$ of treatment with STK899704. These data indicate that STK899704 was not toxic to normal colon cells and colon cancer cells. Next, we conducted phase-contrast microscopy to evaluate the changes in cellular morphology and found that the cell density of HT29 cells and CCD-18Co were not decreased (Fig. 1C). Thus, we used non-cytotoxic concentrations of STK899704 (0.1-0.4 $\mu \mathrm{M})$ in subsequent experiments.

\section{STK899704 inhibited migration of HT29 adherent cells}

To investigate the anti-migration effects of STK899704 on HT29 cells, we performed a wound healing assay and acquired microscopic images of HT29 cells following $48 \mathrm{~h}$ treatment with 0.1-0.4 $\mu \mathrm{M}$ STK899704 treatment. We found that STK899704 treatment reduced the migration of HT29 colon cancer cells into the wounded area (Fig. 2A). Cell motility was enhanced by degradation of the extracellular matrix. Matrix metalloproteinase-2 (MMP-2) and MMP-9 degrade the extracellular matrix and facilitate cell migration (15). Therefore, we investigated the effect of STK899704 on the expression and activity of MMP-2 and MMP-9 by performing qPCR and zymography assays. STK899704 reduced MMP-2 and MMP-9 mRNA expression (Fig. 2B) and secretion (Fig. 2C) in HT29 colon cancer cells. Recent studies showed that aggregated tubulin can induce anti-migration effects by blocking microtubule assembly (16). We investigated the effects of STK899704 on microtubule polymerization by immunofluorescence microscopy. The $\alpha$-tubulin network showed a normal organization in HT29

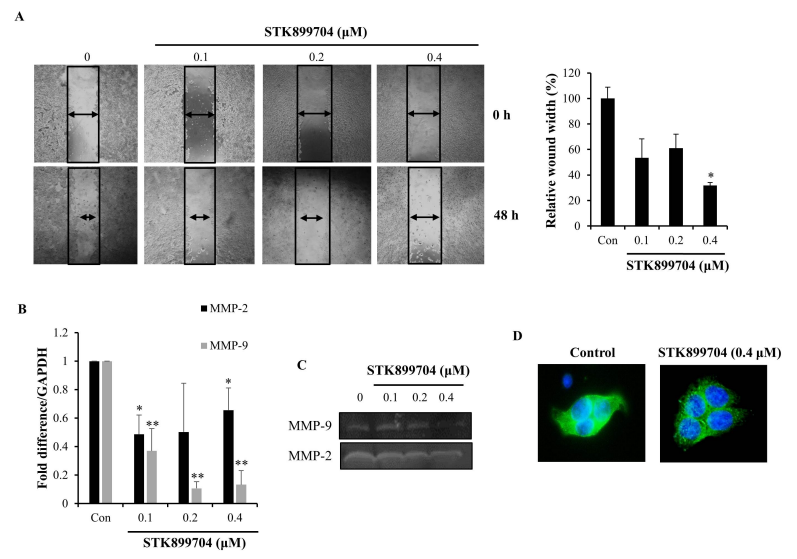

Fig. 2. Inhibitory effect of STK899704 on migration ability of HT29 cells. (A) HT29 cells were treated for $48 \mathrm{~h}$ with STK899704 and cell migration ability was assessed using a wound healing assay. (B) mRNA expression level of MMP-9 and MMP-2 were detected by qPCR in STK899704-treated HT29 cells. (C) Gelatin zymography was performed using the conditioned media of HT29 cells treated with STK899704 and gelatinolytic activity of MMP-9 and MMP-2 were detected. (D) HT29 cells were treated with STK899704 for $24 \mathrm{~h}$. After incubation, the cells were stained with an $\alpha$-tubulin antibody. Cellular microtubules were visualized under a fluorescence microscope. $* \mathrm{P}<0.05$ and $* * \mathrm{P}<0.01$.

cells without STK899704 treatment, while STK899704 treatment resulted in microtubule depolymerization (Fig. 2D). Taken together, these data suggest that STK899704 reduces the migration capacity of HT29 colon cancer cells.

\section{STK899704 inhibited expression of FAK, MEK, and ERK}

Focal adhesion kinase (FAK) is a critical regulator of cancer cell behavior, cell migration, and adhesion. Recent studies suggested that phosphorylation of FAK controls tumor cell adhesion by inducing focal adhesion turnover in colon cancer cells (17). To determine whether STK899704 impedes wound healing through FAK pathway inhibition, we examined the mRNA expression level of $F A K$ by qPCR. FAK expression was decreased in STK899704-treated HT29 cells (Fig. 3A). To further evaluate the regulation of FAK activation by STK899704, we measured FAK phosphorylation levels in immunoblot assays. As shown in Fig. $3 \mathrm{~B}$ and $\mathrm{C}$, FAK phosphorylation was downregulated in HT29 cells treated with STK899704. FAK is linked to the mitogen-activated protein kinase pathway (17). Therefore, we conducted a western blotting assay to determine the effect of STK899704 on mitogen-activated protein kinase kinase (MEK) and extracellular signal-regulated kinase (ERK) protein expression levels in HT29 cells. Fig. 3B and C show that phosphorylated MEK and ERK expression were decreased following STK899704 treatment. Our results suggest that STK899704 inhibited the migration of HT29 cells by downregulating MEK and ERK phosphorylation because of decreased phosphory- 


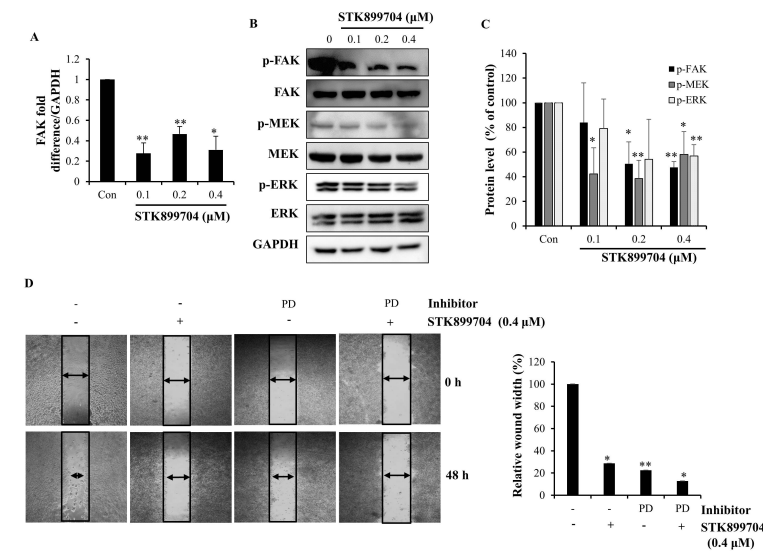

Fig. 3. Inhibitory effect of STK899704 on the expression of FAK, MEK, and ERK and effects on the migration ability of STK899704 and PD98059. (A) Expression of FAK mRNA was analyzed by qPCR. (B) Protein expression levels of p-FAK, p-MEK, and p-ERK were detected by western blot analysis. HT29 cells were treated with the indicated concentration of STK899704 for 24 h. (C) Bar graph represents proteins of p-FAK, p-MEK, and p-ERK. (D) HT29 cells were pretreated with $10 \mu \mathrm{M}$ PD98059 in the absence or presence of $0.4 \mu \mathrm{M}$ STK899704 and cell migration ability was assessed using a wound healing assay. ${ }^{*} \mathrm{P}<0.05$ and ${ }^{*}{ }^{*} \mathrm{P}<0.01$.

lation of FAK. Next, to confirm that STK899704 inhibited this pathway in HT29 colon cancer cells, we treated the cells with an ERK inhibitor (PD98059). As shown in Fig. 3D, the ERK inhibitor (PD98059) inhibited wound closure. Furthermore, co-treatment with STK899704 and ERK inhibitor (PD98059) decreased cell motility also decreased. These results demonstrate that STK899704 decrease the migration ability of HT29 cells through FAK expression.

\section{STK899704 suppressed the properties of HT29 CSCs}

STK899704, a newly identified synthetic compound, has effect on various cancer cells (14), but the potential effects of STK899704 against colon CSCs are not understood. Thus, we investigated whether STK899704 would affect the CSC properties of HT29 and SW620 cells. As shown in Fig. 4A, STK899704 decreased the expression levels of stemnessrelated genes, such as Nanog, Oct4, Sox2, and Bmi1, which were evaluated by qPCR. Based on our results, we further investigated other CSC properties of HT29 and SW620 cells. We induced floating spheroid bodies (18) and examined the spheroid body of CSC. The results showed that STK899704 reduced the diameter and number of spheroid bodies with diameters of $50 \mu \mathrm{m}$ or more for HT29 and SW620 CSCs (Fig. 4B-D). Additionally, STK899704 decreased the number of cells expressing CD133 and CD44, which are used as markers of CSCs in numerous cancers (Fig. 4E, F). Our findings indicate that STK899704 inhibited the expression of stemness genes in CSCs, suggesting that STK899704 suppresses the self-renewal properties of CSCs.

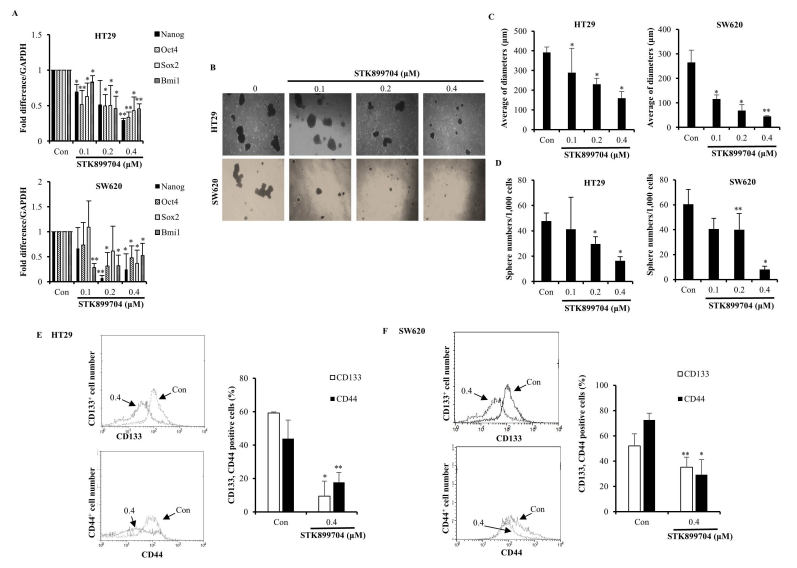

Fig. 4. Inhibitory effect of STK899704 on CSC properties of HT29 and SW620 cells. (A) qPCR assays were conducted to determine the expression level of stemness genes. (B) After CSCs of HT29 and SW620 cells were treated with STK899704, spheroids were observed under a microscope. (C) The average diameters of spheroids were measured. (D) Spheroid bodies of HT29 and SW620 CSCs with a diameter of $\geq 50 \mu \mathrm{m}$ were determined. ( $\mathrm{E}$, F) FACS analysis of HT29 and SW620 CSCs stained with CD133 and CD44 antibody. ${ }^{*} P<0.05$ and $* * P<0.01$.

\section{DISCUSSION}

CSCs have been identified in numerous solid tumors, including breast and melanoma cancers, and were implicated in tumor initiation, metastatic spread, and resistance to anti-cancer therapies (19). Conventional anti-cancer therapies regulate differentiated cancer cells, but not CSCs, and thus alternative strategies are needed to inhibit CSC differentiation and cancer progression. Therefore, novel anti-cancer therapies may facilitate the removal of CSCs.

The compound STK899704 is a novel agent with microtubule-depolymerization activity that binds to tubulin and inhibits its polymerization (14). First, STK899704 did not exhibit cytotoxic effects towards HT29 cells for up to $96 \mathrm{~h}$ (Supplemental Fig. 1). We did not focus on the cytotoxicity or apoptotic effect of STK899704. Because we focused on the migration of cancer cells and stemness of CSCs, we selected a treatment time of $24 \mathrm{~h}$. Our results demonstrated that STK899704 exerted anti-CSC and anti-migration effects in human colon cancer cell lines. The potential effect of microtubule inhibition on cell migration has been investigated through experiments in which treatment with drugs known to interrupt microtubule assembly inhibited cell movement, suggesting cell motility effects via microtubules (20).

In addition to FAK-MEK-ERK signaling, cancer cells use many other mechanisms of migration, such as the PI3K/AKT pathway (21), Notch pathway (22), etc. However, we focused on the FAK-MEK-ERK pathway, which is a strong pathway related to the migration of various cancer cells (23). FAK is 
associated with cell adhesion, proliferation, motility, cancer process and migration (24). Several studies reported hyperphosphorylation, overexpression, and/or increased activity of FAK in numerous human cancers, including astrocytoma, sarcomas, and carcinomas of the colon, liver, and breast (17). Additionally, enhanced FAK activity was found to be associated with metastasis and poorer clinical outcomes (25). This study showed that STK899704 inhibited FAK mRNA expression and phosphorylation of FAK, but not the total FAK protein level (Fig. 3). FAK activates the ERK/mitogen-activated protein kinase cascade (26); thus, we evaluated the relationship between FAK, MEK, and ERK using GIANT and GeneMANIA, and found that the these genes are involved in various pathways, including the actin cytoskeleton regulation pathway (Supplemental Fig. 2).

CD133 and CD44 are glycoproteins and are the most commonly used markers for isolating CSCs including colon cancer, liver cancer, and ovarian cancer (27-30). Our study confirmed that the number of $\mathrm{CD} 133^{+}$and $\mathrm{CD} 44^{+}$cells were decreased in STK899704-treated colon CSCs. Additionally, STK899704-treated colon CSCs showed decreased expression of stemness genes and diameter of spheres in colon CSCs (Fig. 4). These results suggest that STK899704 inhibited the self-renewal properties of colon CSCs. Thus, STK899704 suppressed the CSC properties and migratory behavior of colon cancer cells. Taken together, these data suggest that STK899704 is an efficient therapeutic agent for treating colon cancer.

\section{MATERIALS AND METHODS}

\section{Cell culture}

The HT29 colon cancer cell line was purchased from the American Type Culture Collection (Manassas, VA, USA) and the CCD-18Co normal colon fibroblast cell line was purchased from the Korean Cell Line Bank (Seoul, Korea). The colon cancer cell line was cultured in Dulbecco's modified Eagle's medium (DMEM; HyClone, Logan, UT, USA) and the CCD18-Co cell line was cultured in RPMI-1640 medium (WelGENE, Daegu, Korea) supplemented with 10\% heatinactivated fetal bovine serum (HyClone) at $37^{\circ} \mathrm{C}$ in a $5 \% \mathrm{CO}_{2}$ incubator.

\section{Sphere formation assay}

HT29 cells were seeded $(5,000$ cells $/ \mathrm{ml})$ in ultra-low attachment plates (Corning, Inc., Corning, NY, USA) in tumor sphere medium consisting of DMEM/F12 medium, B27 supplement (1:50), 10\% fetal bovine serum, $10 \mathrm{ng} / \mathrm{ml}$ epidermal growth factor (Calbiochem, San Diego, CA, USA), and $10 \mathrm{ng} / \mathrm{ml}$ basic fibroblast growth factor (Sigma-Aldrich, St. Louis, $\mathrm{MO}, \mathrm{USA}$ ) at $37^{\circ} \mathrm{C}$ in a $5 \% \mathrm{CO}_{2}$ incubator. Every 2-3 days, fibroblast growth factor and epidermal growth factor were added. After 14 days of culture, tumor sphere formation was observed, and the number of tumor spheres formed (diameter $>50 \mu \mathrm{m}$ ) was counted under a microscope.

\section{Reagents}

STK899704 was synthesized at the Korea Research Institute of Bioscience and Biotechnology, and both the detailed synthetic procedure and characterization data were reported previously (14). Its chemical structure is shown in Fig. 1A.

\section{Cell viability assays}

Cell viability was assessed by the MTS (3-[4,5-dimethylthiazol-2yl]-5-[3-carboxy methoxyphenyl]-2-[4-sulfophenyl]-2H-tetrazolium) assay, which measures mitochondrial respiratory function. HT29 cells $\left(3 \times 10^{4}\right.$ cells $\left./ \mathrm{ml}\right)$ were seeded into 96 -well plates, incubated overnight, and treated with various concentrations of STK899704 for 24 h. Cell viability was calculated by assessing MTS metabolism, as previously reported (31). Briefly, media samples $(100 \mu \mathrm{l})$ were removed and incubated with 100 $\mu \mathrm{l}$ of MTS-PMS (phenazine methosulfate) solution for $1 \mathrm{~h}$ at $37^{\circ} \mathrm{C}$. The optical absorbance of the samples was measured at $492 \mathrm{~nm}$ using an enzyme-linked immunosorbent assay reader (Apollo LB 9110, Berthold Technologies GmbH \&Co. KG, Bad Wildbad, Germany).

\section{Gelatin zymography}

HT29 cells $\left(1 \times 10^{5}\right.$ cells/well $)$ were seeded into a 24 -well plate and incubated overnight. Next, the cells were treated with various concentrations of STK899704 for $24 \mathrm{~h}$. The culture supernatant was collected and loaded into a 10\% sodium dodecyl sulfate (SDS)-polyacrylamide gel containing $0.1 \%$ gelatin under non-denaturing conditions to detect MMP-9 and MMP-2. Electrophoresis was performed at a constant $110 \mathrm{~V}$ for $100 \mathrm{~min}$. Gels were washed once with 2.5\% Triton X-100 for 20 min and twice with distilled water for $10 \mathrm{~min}$ at $25^{\circ} \mathrm{C}$, then incubated in zymography reaction buffer at $37^{\circ} \mathrm{C}$ for $18 \mathrm{~h}$. The gels were stained with Coomassie brilliant blue R-250 (Sigma) for $1 \mathrm{~h}$ in a solution of $10 \%$ acetic acid and $10 \%$ methanol and then destained for $1 \mathrm{~h}$.

\section{Wound healing assay}

To evaluate cell motility, HT29 cells $\left(1 \times 10^{5}\right.$ cells/well $)$ were seeded into 24-well plates and incubated overnight at $37^{\circ} \mathrm{C}$ in a $5 \% \mathrm{CO}_{2}$ incubator. Wounds were made using $200-\mu \mathrm{l}$ pipette tips. Cells were washed twice with Hank's balanced salt solution to remove floating cells and fresh medium was added. Cells migrating from the edge of the wound were photographed in five random fields of view at 0 and $48 \mathrm{~h}$ after wounding.

\section{Flow cytometry}

Antibodies against CD133 and CD44 were purchased from Miltenyi Biotec (Bergisch Gladbach, Germany). The expression profiles of CD133 and CD44 in cultured cells were analyzed by flow cytometry. Briefly, HT29 cells were cultured as spheroid bodies. CSCs were dissociated with Accutase ${ }^{\mathbb{R}}$ 
(Sigma) and stained with the CD133 and CD44 antibodies at $37^{\circ} \mathrm{C}$ for 15 min. Populations of $\mathrm{CD}_{133^{+}}$cells and $\mathrm{CD} 44^{+}$ cells were sorted by flow cytometry and the data were analyzed using Cell Quest software (BD Biosciences, Franklin Lakes, NJ, USA).

\section{Reverse transcription polymerase chain reaction analysis} Total RNA was prepared from HT29 and CSCs, using easy-BLUE ${ }^{\mathrm{TM}}$ Total RNA Extraction Kit (iNtRon Biotechnology, Seongnam, Korea) according to the manufacturer's instructions (31). We synthesized cDNA from total RNA samples using M-MuLV reverse transcriptase (New England Biolabs, Ipswich, MA, USA). Quantitative reverse transcription polymerase chain reaction (qPCR) was performed with Roter-Gene 6,000 series software 1.7 (Qiagen, Hilden, Germany) and the SensiFASTTM SYBR NO-ROX Kit (BIOLINE, London, UK). We used oligonucleotide primer sequences specific for Oct4 (5'-GGT TCT CGA TAC TGG TTC GC-3' and 5'-GTG GAG GAA GCT GAC AAC AA-3'), Sox2 (5'-GCT TAG CCT CGT CGA TGA AC-3' and 5'-AAC CCC AAG ATG CAC AAC TC-3'), Bmi1 (5'-AAA TGC TGG AGA ACT GCA AAG-3' and 5' CTG TGG ATG AGG AGA CTG C-3'), Nanog (5'-ATG GAG GGA AGA GGA GA-3' and 5'-GAT TTG TGG GCC TGA AGA AA-3'), MMP-9 (5'-GCC ATT CAC GTC CTT AT-3' and 5'-TTG ACA GCG ACA AGA AGT GG-3'), MMP-2 (5'-CAA AGG GGT ATC CAT CGC CA-3' and 5'-CGC CCC CAA AAC GGA CAA AG-3'), FAK (5'-AGT AAA ATC CAG CCA GCC CC-3' and 5'-GCT CAC CCA GGT CAG AGT TC-3'), and GAPDH (5'-TGG GCT ACA CTG AGC ACC AG-3' and 5'-GGG TGT CGT TGT AGT CA-3'). The relative gene expression differences were calculated using threshold cycle (CT) values which were normalized to the GAPDH gene as an internal control in the same sample.

\section{Western blot analysis}

Cells were treated with STK899704, harvested, and lysed in buffer containing $50 \mathrm{mM}$ Tris $(\mathrm{pH}$ 7.4), $1.5 \mathrm{M}$ sodium chloride, $1 \mathrm{mM}$ ethylenediaminetetraacetic acid, 1\% NP-40, $0.25 \%$ sodium deoxycholate, $0.1 \%$ SDS, and a protease inhibitor cocktail. Lysates were boiled at $100^{\circ} \mathrm{C}$ for $5 \mathrm{~min}$. Equal amounts of total proteins were resolved by $10-12 \%$ SDS-polyacrylamide gel electrophoresis. Proteins were transferred onto polyvinylidene difluoride membranes (Millipore, Billerica, MA, USA) and incubated overnight at $4^{\circ} \mathrm{C}$ with specific primary antibodies against p-MEK [Ser217/221], p-ERK [Thr202/Tyr204], MEK, ERK, FAK (Cell Signaling Technology, Danvers, MA, USA), p-FAK [Y397] (Invitrogen, Carlsbad, CA, USA), and GAPDH. Membranes were washed with Trisbuffered saline containing $0.05 \%$ Tween 20 and incubated with secondary antibodies (horseradish peroxidase-conjugated anti-rabbit or anti-mouse IgG) for $1 \mathrm{~h}$ at room temperature. Specific protein bands were detected with the West-Zol Plus western blot detection system (iNtRON Biotechnology).

\section{Immunofluorescence}

Immunofluorescence assays were performed as previously described (32). Briefly, cells seeded onto glass coverslips and incubated overnight at $37^{\circ} \mathrm{C}$ in a $5 \% \mathrm{CO}_{2}$ incubator, followed by treatment with STK899704. After $24 \mathrm{~h}$, the cells were permeabilized, fixed with $100 \%$ acetone, and blocked with $0.1 \%(\mathrm{w} / \mathrm{v})$ bovine serum albumin. The cells were incubated overnight with antibodies against $\alpha$-tubulin at room temperature. Following incubation, the cells were treated with secondary anti-mouse antibodies for $1 \mathrm{~h}$ at room temperature. Fluorescence images were obtained using a BX61-32FDIC (Olympus, Tokyo, Japan) upright fluorescence microscope.

\section{Analysis of Gene Network}

GIANT (Genome-scale Integrated Analysis of gene Networks in Tissues) was applied to perform a network analysis of the various genes. GIANT analyzes the relationships between a set of input genes and predicted genes. GIANT is available at http://giant.princeton.edu/. GeneMANIA was used for gene interaction analysis. The dataset is available at http://www. genemania.org.

\section{Statistical analyses}

Statistically significant differences were determined by Student's $t$-test. Data are expressed as the means \pm standard error of the mean. A value of $P<0.05$ was considered significant, while $\mathrm{P}<0.01$ was considered very significant.

\section{ACKNOWLEDGEMENTS}

This research was supported by the basic program (2018R1A 2B2001225) of the National Research Foundation of Korea (NRF).

\section{CONFLICTS OF INTEREST}

The authors have no conflicting interests.

\section{REFERENCES}

1. Siegel R, Naishadham D and Jemal A (2012) Cancer statistics for Hispanics/Latinos, 2012. CA Cancer J Clin 62, 283-298

2. Lozano R, Naghavi M, Foreman K et al (2012) Global and regional mortality from 235 causes of death for 20 age groups in 1990 and 2010: a systematic analysis for the global burden of disease study 2010. Lancet 380, 2095-2128

3. Jemal A, Bray F, Center MM, Ferlay J, Ward E and Forman D (2011) Global cancer statistics. CA Cancer J Clin 61, 69-90

4. Bak Y, Kwon T, Bak IS, Hong J, Yu DY and Yoon DY (2016) IL-32theta inhibits stemness and epithelialmesenchymal transition of cancer stem cells via the STAT3 pathway in colon cancer. Oncotarget 7, 7307- 
7317

5. Merika E, Saif MW, Katz A, Syrigos K and Morse M (2010) Review. Colon cancer vaccines: an update. In Vivo 24, 607-628

6. Bonnet D and Dick JE (1997) Human acute myeloid leukemia is organized as a hierarchy that originates from a primitive hematopoietic cell. Nat Med 3, 730-737

7. Jordan CT (2009) Cancer stem cells: controversial or just misunderstood? Cell Stem Cell 4, 203-205

8. Lessard J and Sauvageau G (2003) Bmi-1 determines the proliferative capacity of normal and leukaemic stem cells. Nature 423, 255-260

9. Lee HE, Kim JH, Kim YJ et al (2011) An increase in cancer stem cell population after primary systemic therapy is a poor prognostic factor in breast cancer. Br J Cancer 104, 1730-1738

10. Gao XM, Zhang R, Dong QZ and Qin LX (2016) Properties and feasibility of using cancer stem cells in clinical cancer treatment. Cancer Biol Med 13, 489-495

11. Vinogradov S and Wei X (2012) Cancer stem cells and drug resistance: the potential of nanomedicine. Nanomedicine (Lond) 7, 597-615

12. Jordan MA and Wilson $L$ (2004) Microtubules as a target for anticancer drugs. Nat Rev Cancer 4, 253-265

13. Zhou J and Giannakakou P (2005) Targeting microtubules for cancer chemotherapy. Curr Med Chem Anticancer Agents 5, 65-71

14. Sakchaisri K, Kim SO, Hwang J et al (2017) Anticancer activity of a novel small molecule tubulin inhibitor STK899704. PLoS One 12, e0173311

15. Gialeli C, Theocharis AD and Karamanos NK (2011) Roles of matrix metalloproteinases in cancer progression and their pharmacological targeting. FEBS J 278, 16-27

16. Liang W, Lai $Y$, Zhu M, Huang S, Feng $W$ and Gu X (2016) Combretastatin A4 regulates proliferation, migration, invasion, and apoptosis of thyroid cancer cells via PI3K/Akt signaling pathway. Med Sci Monit 22, 4911-4917

17. McLean GW, Carragher NO, Avizienyte E, Evans J, Brunton VG and Frame MC (2005) The role of focal-adhesion kinase in cancer - a new therapeutic opportunity. Nat Rev Cancer 5, 505-515

18. Pastrana E, Silva-Vargas $V$ and Doetsch F (2011) Eyes wide open: a critical review of sphere-formation as an assay for stem cells. Cell Stem Cell 8, 486-498

19. Al-Hajj M, Wicha MS, Benito-Hernandez A, Morrison SJ and Clarke MF (2003) Prospective identification of tumorigenic breast cancer cells. Proc Natl Acad Sci U S A 100, 3983-3988

20. Goldman RD (1971) The role of three cytoplasmic fibers in BHK-21 cell motility. I. Microtubules and the effects of colchicine. J Cell Biol 51, 752-762

21. Han YS, Lee JH and Lee SH (2015) Fucoidan inhibits the migration and proliferation of HT-29 human colon cancer cells via the phosphoinositide-3 kinase/Akt/mechanistic target of rapamycin pathways. Mol Med Rep 12, 3446-3452

22. Mu Y, Zou H, Chen B, Fan Y and Luo S (2017) FAM83D knockdown regulates proliferation, migration and invasion of colorectal cancer through inhibiting FBXW7/Notch-1 signalling pathway. Biomed Pharmacother 90, 548-554

23. Wang YJ, Zhang ZF, Fan SH et al (2017) MicroRNA-433 inhibits oral squamous cell carcinoma cells by targeting FAK. Oncotarget 8, 100227-100241

24. Deramaudt TB, Dujardin D, Noulet F et al (2014) Altering FAK-paxillin interactions reduces adhesion, migration and invasion processes. PLoS One 9, e92059

25. Pylayeva Y, Gillen KM, Gerald W, Beggs HE, Reichardt LF and Giancotti FG (2009) Ras- and PI3K-dependent breast tumorigenesis in mice and humans requires focal adhesion kinase signaling. J Clin Invest 119, 252-266

26. Schlaepfer DD, Mitra SK and Ilic D (2004) Control of motile and invasive cell phenotypes by focal adhesion kinase. Biochim Biophys Acta 1692, 77-102

27. Suva ML, Riggi N, Stehle JC et al (2009) Identification of cancer stem cells in Ewing's sarcoma. Cancer Res 69, 1776-1781

28. Suetsugu A, Nagaki M, Aoki $H$, Motohashi T, Kunisada $T$ and Moriwaki H (2006) Characterization of CD133+ hepatocellular carcinoma cells as cancer stem/progenitor cells. Biochem Biophys Res Commun 351, 820-824

29. Hurt EM, Kawasaki BT, Klarmann GJ, Thomas SB and Farrar WL (2008) CD44 + CD24(-) prostate cells are early cancer progenitor/stem cells that provide a model for patients with poor prognosis. Br J Cancer 98, 756-765

30. Kim WT and Ryu CJ (2017) Cancer stem cell surface markers on normal stem cells. BMB Rep 50, 285-298

31. Bak Y, Ham S, Baatartsogt O et al (2013) A1E inhibits proliferation and induces apoptosis in $\mathrm{NCl}-\mathrm{H} 460$ lung cancer cells via extrinsic and intrinsic pathways. Mol Biol Rep 40, 4507-4519

32. Bak Y, Kang JW, Kim MS et al (2014) IL-32theta downregulates CCL5 expression through its interaction with PKCdelta and STAT3. Cell Signal 26, 3007-3015 